Published online on the journal's webpage: http://jurnal.iaii.or.id

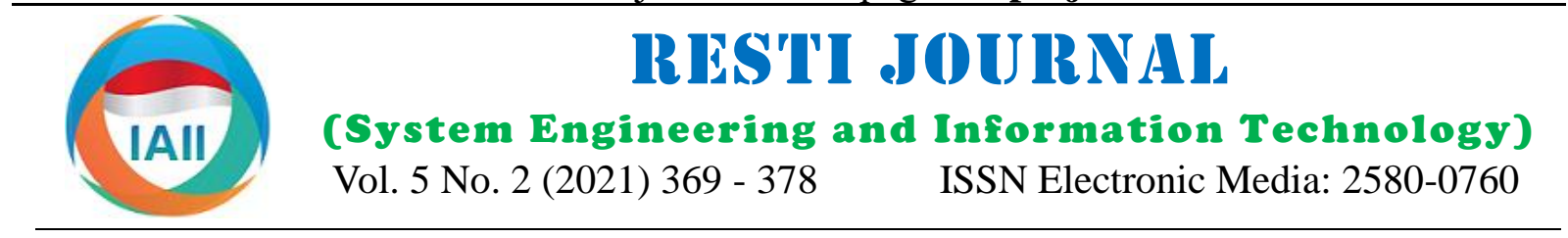

\title{
Random Forest Algorithm to Investigate the Case of Acute Coronary Syndrome
}

\author{
Eka Pandu Cynthia ${ }^{1}$, M. Afif Rizky A. ${ }^{2}$, Alwis Nazir ${ }^{3}$, Fadhilah Syafria ${ }^{4}$ \\ 1,2,3,4 Teknik Informatika, Fakultas Sains dan Teknologi, UIN Sultan Syarif Kasim Riau \\ 11eka.pandu.cynthia@uin-suska.ac.id, ${ }^{2}$ afifrizky933@ gmail.com, ${ }^{3}$ alwis.nazir@uin-suska.ac.id, ${ }^{4}$ fadhilah.syafria@uin- \\ suska.ac.id
}

\begin{abstract}
This paper explains the use of the Random Forest Algorithm to investigate the Case of Acute Coronary Syndrome (ACS). The objectives of this study are to review the evaluation of the use of data science techniques and machine learning algorithms in creating a model that can classify whether or not cases of acute coronary syndrome occur. The research method used in this study refers to the IBM Foundational Methodology for Data Science, include: i) inventorying dataset about ACS, ii) preprocessing for the data into four sub-processes, i.e. requirements, collection, understanding, and preparation, iii) determination of RFA, i.e. the " $\mathrm{n}$ " of the tree which will form a forest and forming trees from the random forest that has been created, and iv) determination of the model evaluation and result in analysis based on Python programming language. Based on the experiments that the learning have been conducted using a random forest machine-learning algorithm with an n-estimator value of 100 and each tree's depth (max depth) with a value of 4, learning scenarios of 70:30, 80:20, and 90:10 on 444 cases of acute coronary syndrome data. The results show that the 70:30 scenario model has the best results, with an accuracy value of $83.45 \%$, a precision value of $85 \%$, and a recall value of $92.4 \%$. Conclusions obtained from the experiment results were evaluated with various statistical metrics (accuracy, precision, and recall) in each learning scenario on 444 cases of acute coronary syndrome data with a cross-validation value of 10 fold.
\end{abstract}

Keywords: artificial intelligence, data processing, machine learning, random forest algorithm, supervised learning.

\section{Introduction}

Previous studies that used the random forest algorithm in solving health cases, such as the detection of congestive heart failure using ECG waves using the random forest algorithm where the study was an experiment that produced various statistical measures against the use of the random forest algorithm for the case of detection of congestive heart failure. From these studies, it can be concluded that the random forest algorithm produces a performance that is considered significant in detecting the disease being studied and provides an insight into expressing cases of congestive heart failure using ECG with $100 \%$ accuracy [1]. The random forest algorithm is also used in breast cancer This algorithm generates a random decision tree. The detection, where this study uses a digital track record of random forest will select the best tree and will be issued 699 instances of 10 attributes. From these studies, it can as a decision tree. The random forest algorithm has been be concluded that the random forest algorithm provides widely researched and applied in the health sector. Alam a significant performance compared to the conventional et al. presented a random-forest-based predictor for random binary classifier method with a correlation medical data classification using feature ranking [8]. accuracy of $97 \%$ and a sensitivity of $96 \%$ [2]. Byeon Kaur et al. combined the random forest algorithm and conducted a study proving whether the RFA is suitable the internet of things (IoT) in a health monitoring system 
[9]. Ricordeau et al. applied the random forest algorithm Based on the background and various studies conducted to a health monitoring machine [10]. Kumar performed in the health sector using the machine learning a health care analysis using the random forest algorithm algorithms that have been mentioned, especially in [11]. Oliver Pauly has presented in his thesis the research using the RFA, the author will conduct research application of the random forest algorithm in several and analysis using data science techniques to produce a medical applications such as Multiple Organ Detection model using the RFA against cases of the ACS. This and Localization in multi-channel Magnetic Response study can find various information, patterns, knowledge, scans, Multiple Organ Segmentation in CT scans, and and performance of the random forest algorithm in Detection of Substantia Nigra Echogenicities in 3D classifying whether or not cases of ACS occur.

Transcranial Ultra-sound. Towards Computer-Aided Diagnosis of Parkinson Disease [12].

\section{Research Method}

Data science, machine learning, and artificial This research was conducted by building a model that intelligence are becoming top-trending topics in today's can classify ACS into two classes: positive ACS and technological world. It is a common thing because today negative ACS, without using data normalization with a and in the future, the world has entered the era of big random forest classification algorithm. The following is data [13]. In processing such a lot of data, data science the modeling workflow built-in performing the ACS is the most sought-after scientific field in the period of classification. The flowchart for research methods is big data like now. It happens because data science is a shown in Figure 1.

data processing science. Data science is not only the science of data processing, but data science functions to combine various techniques, algorithms, and principles of machine learning that are useful for finding hidden patterns in data [14]. Machine Learning is an essential topic in line with the development of knowledge and businesses looking for innovative ways to support and sustain the business realm in reaching new understanding levels [15]. It is because machine learning can help agencies and organizations have the ability to predict what will happen next. A data scientist can process data to find various clustering patterns, regression, and classification to obtain data. It can be seen what patterns, information, and knowledge can help problems such as forecasting, time series, business intelligence, data analytics, and research: prediction, sentiment analysis, and various other practitioners [16].

Data science can determine the paradigm of health cases with multiple techniques and tools in machine learning, statistics, and data visualization in the health sector. In the health sector's case, best practice data science recognizes symptoms, risks and recommends planning before these health risks occur to a person [17]. With data science, a data scientist can get a pattern based on modeling health cases and provide various visualizations for others' knowledge that can be shared with others. The World Health Organization (WHO) states that acute heart disease or coronary syndrome is currently one of the causes of the highest death cases. Every year in the world, it shows that 17.9 million people (31\% of global deaths) are caused by coronary heart disease [18]. Akyol et al. have analyzed the Demographic Characteristics of Making Coronary Artery Disease Susceptible using the Random Forests Classifier [19]. Yekkala et al. Combined the random forest algorithm and selection features in predicting heart disease [20]. Ani et al. used the Random Forest Ensemble Classifier to Predict Coronary Heart Disease Using Risk Factors [21].

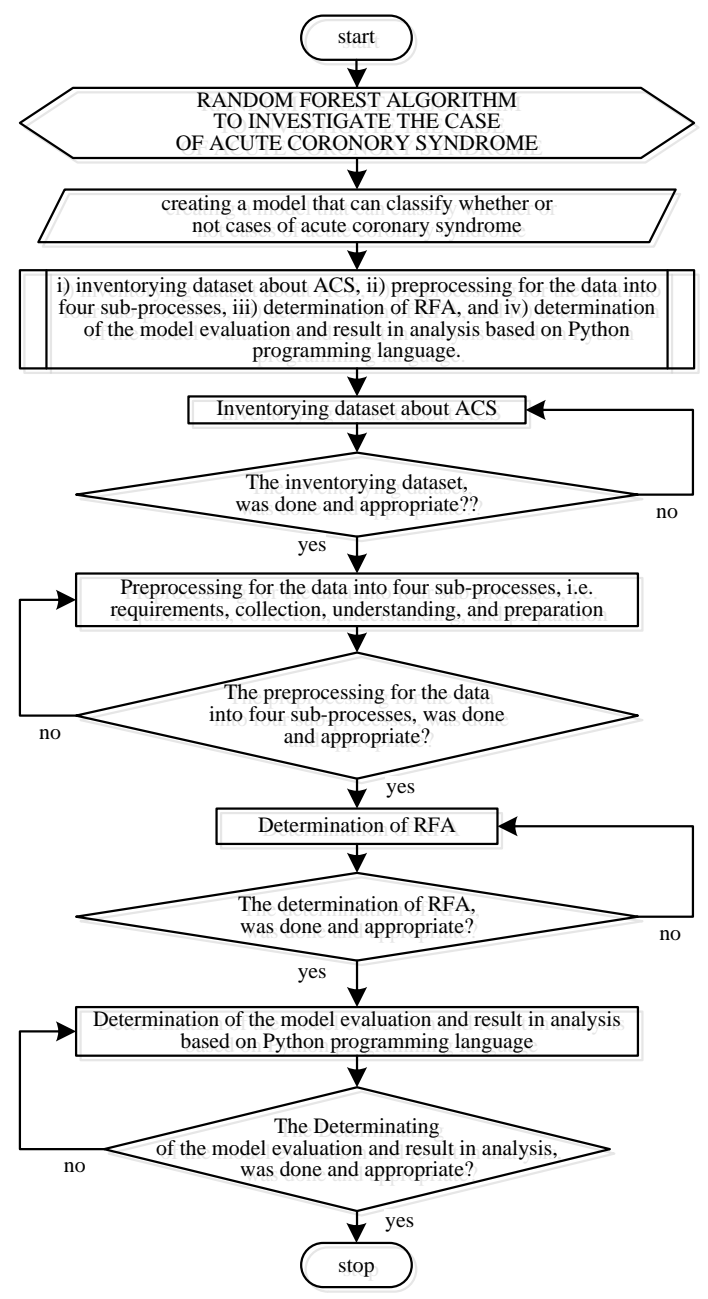

Figure 1. The flowchart for research methods

Based on Figure 1 it can be explained, that the implementation of the research methods in four stages, include i) inventorying dataset about ACS, ii) preprocessing for the data into four sub-processes, i.e. 
requirements, collection, understanding, and Sp.An and general practitioner, dr. Alfarisy Zamzami preparation, iii) determination of RFA, i.e. the " $n$ " of the regarding the required parameters. The data used in this tree which will form a forest and forming trees from the study were diagnostic data for patients with an acute random forest that has been created, and iv) coronary syndrome which came from two different determination of the model evaluation and result in sources, namely 303 data from Medan and 141 medical analysis based on Python programming language.

\subsection{Inventorying Dataset about ACS} records from Arifin Achmad Hospital Pekanbaru. The data used consists of 13 input parameters that represent cases of acute coronary syndrome by cardiac Before data collection is carried out, it will first require anesthetists, and the data is more towards laboratory data data specifications suitable for research. It is done by with the following information. The description of each conducting a short interview with a cardiac data parameter is shown in Table 1 anesthesiologist, namely dr. Vera Muharrami, M.Ked.,

Table 1. The description of each data parameter

\begin{tabular}{|c|c|c|}
\hline No. & Parameter Name & Information \\
\hline 1 & Age & Patient's Age \\
\hline 2 & Gender & Patient's Gender \\
\hline 3 & Angina's Type & Description of the type and pain in the chest experienced by the patient \\
\hline 4 & Resting Blood Pressure & Blood pressure measurements are taken when the patient is resting / not doing activities \\
\hline 5 & Cholesterol & Measurement of the patient's cholesterol \\
\hline 6 & Fasting/Time Blood Sugar & Blood sugar measurements are taken when the patient is ordered to fast by a specialist \\
\hline 7 & Electrocardiography Waveform & The shape of the wave size when the patient is placed on an electrocardiography device \\
\hline 8 & Maximum Heart Rate & A sampling of the patient's maximum heart rate \\
\hline 9 & Angina Activities & Patient information regarding the presence of chest pain during activities \\
\hline 10 & ST_T ECG Line Length & Measure the length of the ST_T line from the ECG patient medical record paper \\
\hline 11 & ST_T Slope ECG Shape & Assess the $\mathrm{ST}_{-} \mathrm{T}$ Slope shape of cardiac patients \\
\hline 12 & Num major vessels & Enlarged size of the patient's large blood vessels \\
\hline 13 & Thalassemia & A congenital blood disorder characterized by a lack of oxygen-carrying proteins \\
\hline
\end{tabular}

\subsection{Preprocessing}

essential parameters in acute coronary syndrome, as

In this study, the preprocessing stage was divided into shown in Table 1.

four sub-processes, i.e. the data requirements stage, the All writing of each parameter above was done with a data collection stage, the data understanding or cardiac anesthetist specialist at RSUD Arifin Achmad exploratory data analysis (EDA) stage, and the data Pekanbaru. After the data is collected, a table is preparation stage. The data requirement stage is how to compiled in a Comma Separated Value (CSV). The EDA determine the correct data specifications to be used in stage is an essential part of data science, which means research. In this study, the required data specifications that we must understand how the data composition is are medical records of acute coronary syndrome patients seen in terms of data distribution and statistics so that we in inpatient rooms or CVCU rooms. This data is needed can get an idea of what is happening in the data. The because it has a history more complete than outpatient EDA process for acute coronary syndrome case data data. At the data collection stage, was collect all includes import Python Library, read data, statistics specified data to obtain various information such as description, and analysis. The results obtained in the parameter requirements and values used during the statistical process describe and analyze. The showing of research. Based on the medical records obtained, there five variables with statistic description and analysis is are different parameters available but limited to 13 shown in Figure 2.

\begin{tabular}{crrrrr} 
& age & gender & cholesterol & resting_blood_pressure & maximum_heart_rate \\
\hline count & 444.000000 & 444.000000 & 444.000000 & 444.000000 & 444.000000 \\
mean & 53.416667 & 0.662162 & 217.085586 & 124.441441 & 133.768018 \\
std & 11.933404 & 0.473507 & 65.424827 & 20.944271 & 31.178692 \\
min & 0.000000 & 0.000000 & 71.000000 & 62.000000 & 40.000000 \\
$\mathbf{2 5} \%$ & 46.000000 & 0.000000 & 170.750000 & 110.000000 & 105.750000 \\
$\mathbf{5 0} \%$ & 55.000000 & 1.000000 & 216.500000 & 125.000000 & 138.500000 \\
$\mathbf{7 5} \%$ & 61.000000 & 1.000000 & 259.250000 & 138.000000 & 160.250000 \\
max & 88.000000 & 1.000000 & 564.000000 & 200.000000 & 202.000000
\end{tabular}

Figure 2. The showing of five variables with statistic description and analysis

DOI: https://doi.org/10.29207/resti.v5i2.3000

Creative Commons Attribution 4.0 International License (CC BY 4.0) 
The EDA process can also provide visualization related seen from the results above, patients with average to the distribution of data and statistical values that exist cholesterol had a lesser frequency of 171 cases than 273 in the data. Figure 3 below is an example of a patients with cholesterol above $200 \mathrm{mg} / \mathrm{dL}$. The curve of visualization of cholesterol distribution. Normal a cholesterol distribution visualization is shown in cholesterol, as we know, it is <200 mg / dL. However, if Figure 3.

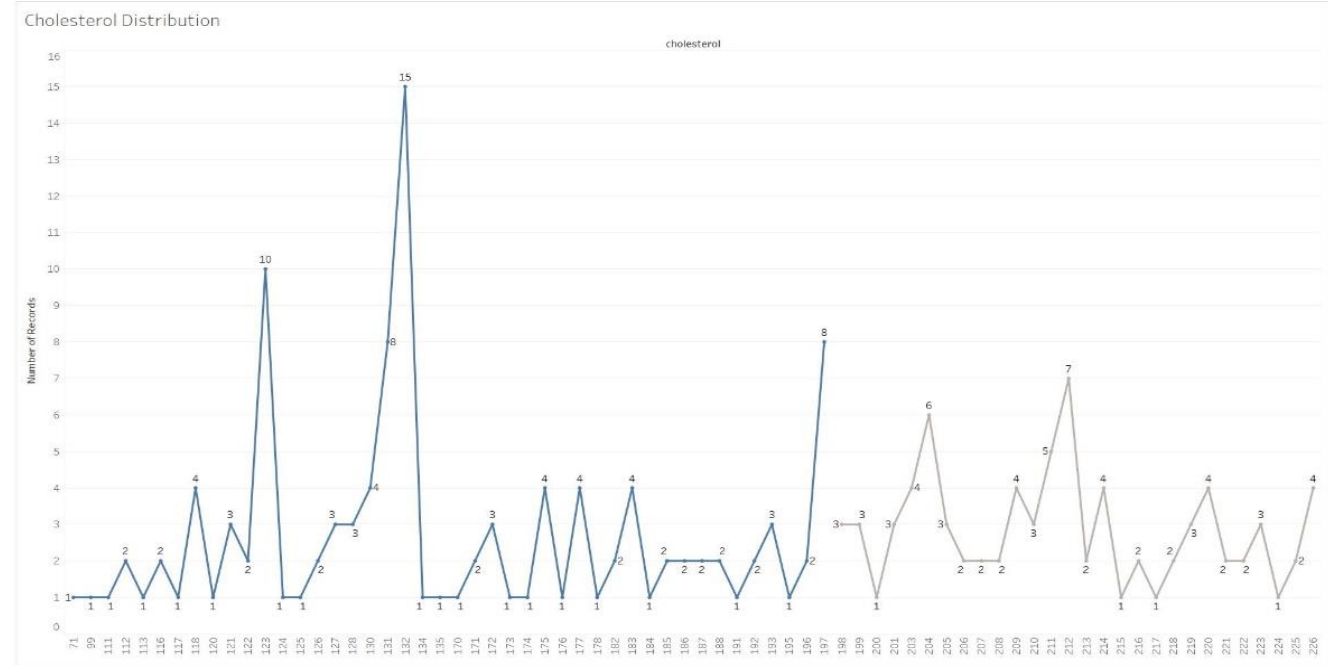

Figure 3. The curve of a cholesterol distribution visualization

Next, we visualize the correlation matrix between have cases than other variables. Based on these variables. In this visualization, we can translate more visualization results, there are no variables that are zero deeply about what happens to the data we have. For or do not correlate at all with other variables, so it can be example, the correlation between age and target is concluded that all variables are important compositions negative -0.18 , which means that the lower the age, the of acute coronary syndrome cases and will be used for less likely a person is to develop the acute coronary the modeling process using the random forest algorithm. syndrome. Meanwhile, gender also has a negative The visualization of the correlation matrix between correlation, which means that women are less likely to variables is shown in Figure 4.

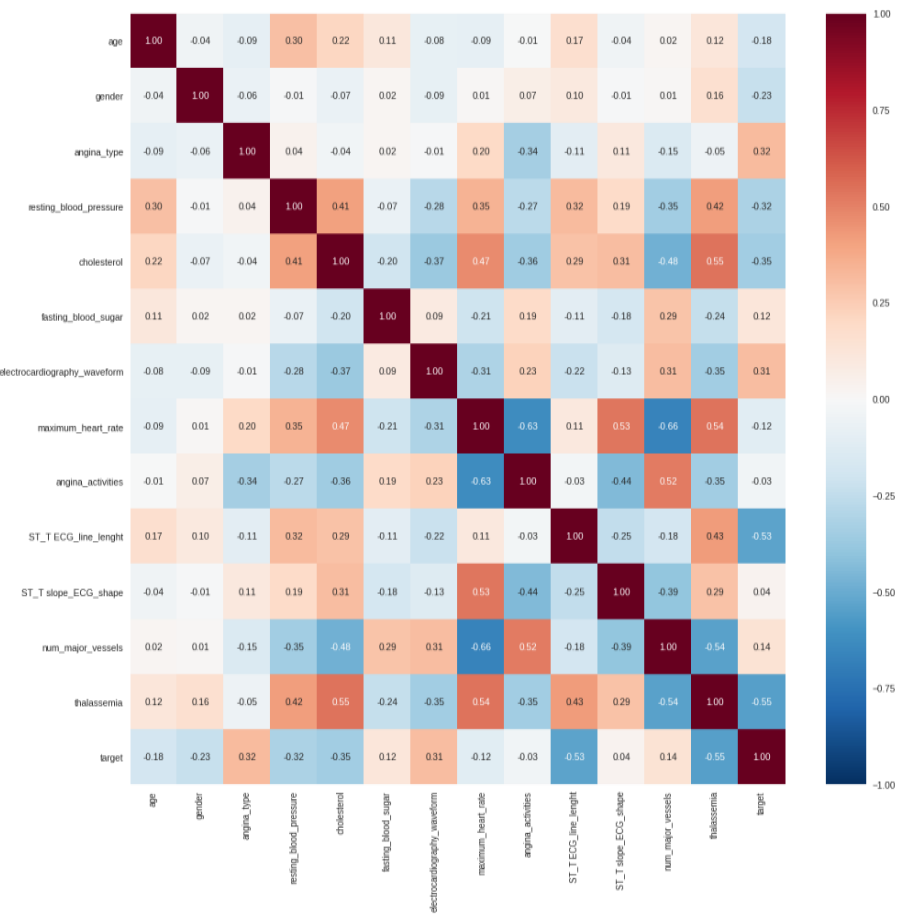

Figure 4. The visualization of correlation matrix between variables

DOI: https://doi.org/10.29207/resti.v5i2.3000 
In the data preparation stage, the extract of parameters from the data used, namely the data type transformation, making the data easier to understand and train test split data. At the stage of parameter and data transformation, first, we change the data so that it is easier to understand. Second, the categorical parameter is changed from int64 to object. It is also done to make it easier for the algorithm to read the data type when it becomes the random forest algorithm's input. Third, we create a dummies variable that indicates and separates a variable with categorical values into a unique value. The transformation process can be seen in the following code listing, i.e.:

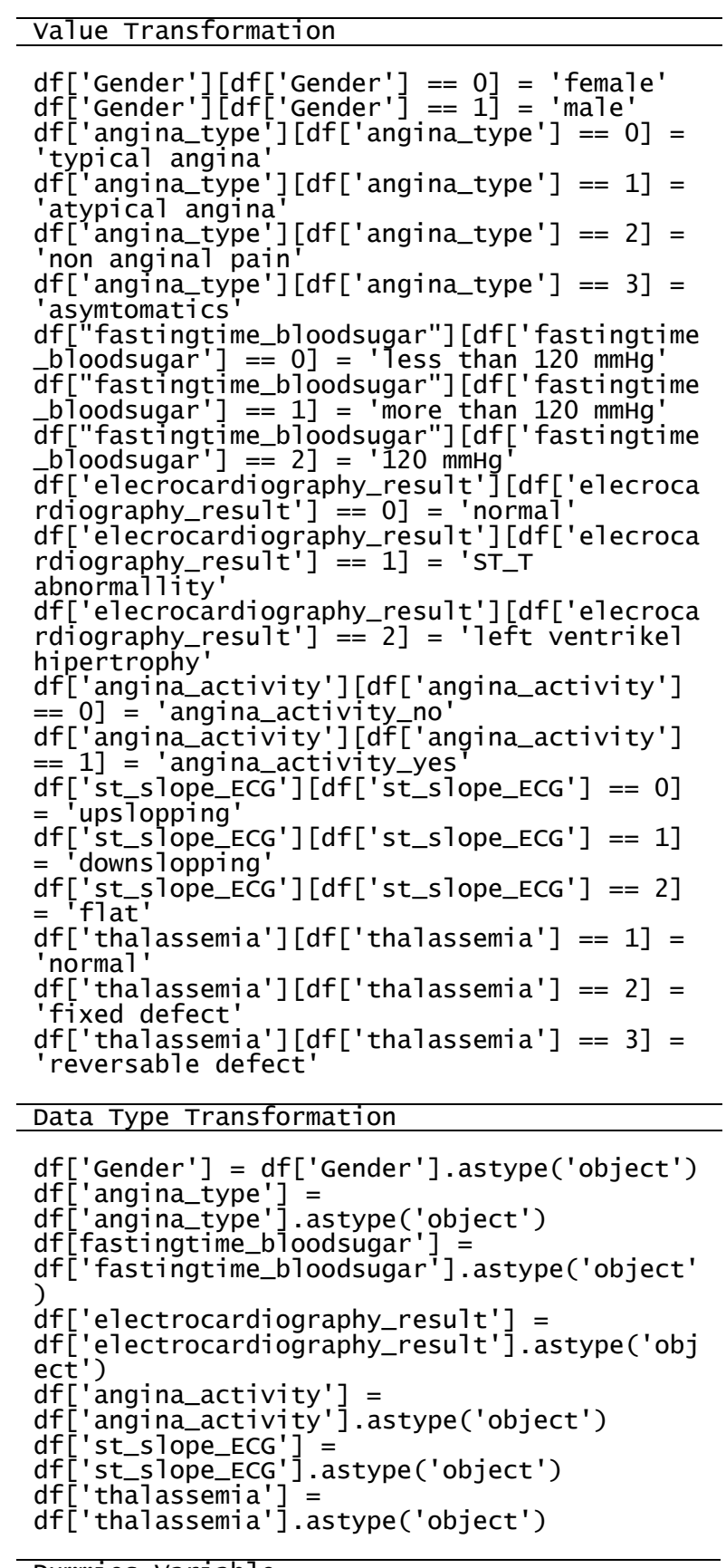

df = pd.get_dummies(df, drop_first=True)

The next stage will separate the training data (train data) and test data (test data). Data split is carried out in sizes of 0.2 (80\% training data, $20 \%$ testing data), $0.3(70 \%$ training data, 30\% testing data), and 0.1 (90\% training data, $10 \%$ testing data). Data sharing is done using the stratified method, which means that the y_value (target class) condition is the same as the magnitude or proportion of the data separation condition. The separation of training and test data is shown in Table 2.

Table 2. The separation of training and test data

\begin{tabular}{ccccccc}
\hline $\begin{array}{c}\text { Split } \\
\text { Model }\end{array}$ & $\begin{array}{c}\text { Amount } \\
\text { of Data }\end{array}$ & Class 0 & Class 1 & $\begin{array}{c}\text { Split } \\
\text { Train: } \\
\text { Test }\end{array}$ & $\begin{array}{c}\text { Y_Train } \\
\text { Stratify }\end{array}$ & $\begin{array}{c}\text { Data } \\
\text { Testing }\end{array}$ \\
\hline $70 \%$ & 444 & 138 & 306 & $310: 133$ & $213: 97$ & $92: 41$ \\
$80 \%$ & 444 & 138 & 306 & $354: 89$ & $244: 110$ & $61: 28$ \\
$90 \%$ & 444 & 138 & 306 & $398: 45$ & $274: 124$ & $31: 14$ \\
\hline
\end{tabular}

\subsection{Determinating of RFA}

The next step is to explain the random forest algorithm's flow in processing acute coronary syndrome data. The random forest algorithm is divided into two parts. The first part is the "n" (tree) which will form a forest with random values. The second part is the algorithm for forming trees from the random forest that has been created.

Input:

\section{$D$, a dataset consisting of $d$ rows \\ $k$, the number from the number of trees}

The Random Forest method [22] first created a sample data by taking random from dataset $\mathrm{D}$ with replacement. Second, use the Di sample data to build the ith tree $(\mathrm{i}=$ $1,2, \ldots k)$, and third, repeat steps one and two for $k$.

The random forest algorithm begins with the initial selection of " $\mathrm{k}$ " samples from the data set, which is done randomly (random). Furthermore, based on the initiation, it is used to form each tree independent from the other trees. After the tree has been built to completion, each tree will give each tree a majority vote against the tree that has received the most votes from the other trees. It makes the tree contained in the random forest algorithm untraceable because it uses a random subset of the existing dataset. The flowchart of the Random Forest algorithm is shown in Figure 5.

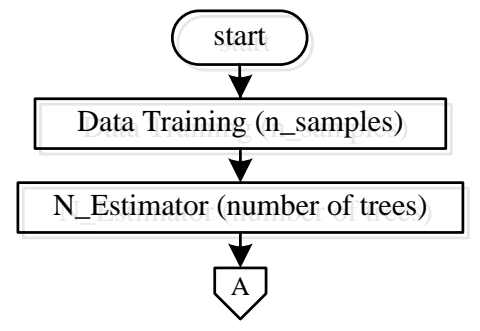




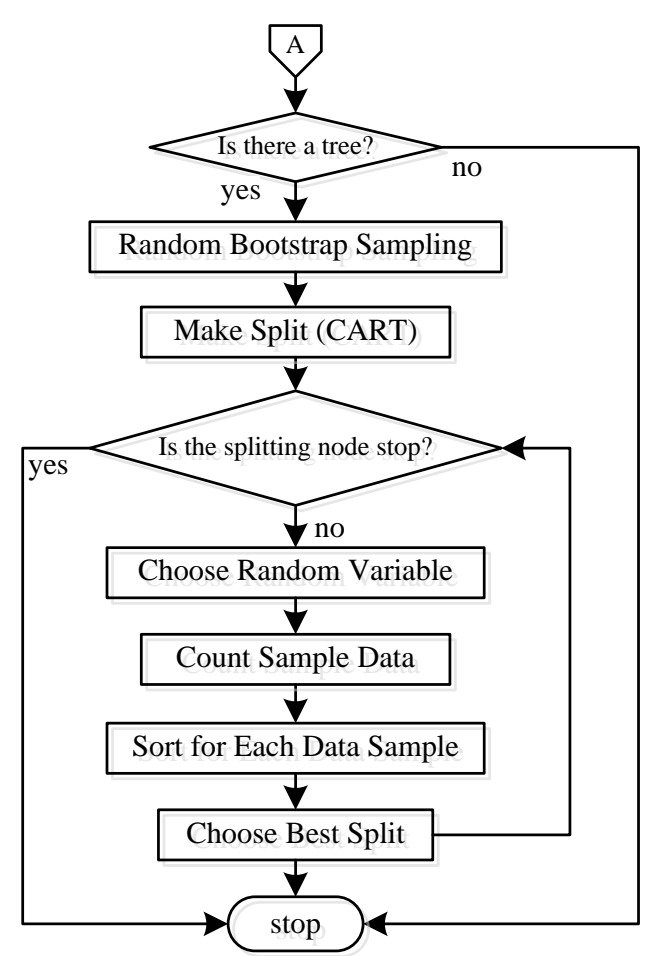

Figure 5. The flowchart of the Random Forest algorithm

\subsection{Model Evaluation and Result Analysis}

This study uses the Python programming language parameters in the dataset of acute coronary syndrome version 3.7.4 and the Integrated Development cases are carried out in the data preprocessing stage, Environment (IDE) jupyter which can be seen in section 2.2 to make the data easier notebook/jupyterlab/googlecollab. Model evaluation to process or use in data mining. The following is patient Analysis of the results of modeling using the random data on acute coronary syndrome cases used in forest algorithm in cases of the acute coronary syndrome modeling. The patient data on acute coronary syndrome is carried out in several stages: First, using the Confusion cases are shown in Table 4.

Matrix test [23], which is used to calculate the level of accuracy described in the table which states the number of correct test data and incorrectly classified test data. Confusion matrix testing is done based on a data set that has two classes, namely positive class, and negative class. Different classes consist of four cells, namely True Positive (TP), False Positive (FP), True Negative (TN), and False Negative (FN). Based on these terms, it can be described in confusion matrix. The confusion matrix is shown in Table 3.

Table 3. The confusion matrix

\begin{tabular}{llll}
\hline \multirow{2}{*}{ Classification } & & \multicolumn{2}{c}{ Result Prediction Class } \\
& & Yes & No \\
\hline \multirow{2}{*}{ Actual Class } & Yes & TP & FN \\
& No & FP & TN \\
\hline
\end{tabular}

The classification model performance level can be measured and evaluated by calculating the accuracy from the confusion matrix table. Accuracy is the percentage of test data that can be classified correctly by the built classification model. The equation of accuracy [23] is shown in Eq. (1).
Table 4. The patient data on acute coronary syndrome cases

\begin{tabular}{lcccc}
\hline \multicolumn{1}{c}{ Parameter's Name } & Patient's & Medical & Record & Value \\
\hline Age & 63 & 37 & 41 & $\ldots$ \\
Gender & 1 & 1 & 0 & $\ldots$ \\
Angina's Type & 3 & 2 & 1 & $\ldots$ \\
Resting Blood Pressure & 145 & 130 & 130 & $\ldots$ \\
Cholesterol & 233 & 250 & 204 & $\ldots$ \\
Fasting/Time Blood Sugar & 1 & 0 & 0 & $\ldots$ \\
Electrocardiography & 0 & 1 & 0 & $\ldots$ \\
Waveform & & & & \\
Maximum Heart Rate & 150 & 187 & 172 & $\ldots$ \\
Angina activities & 0 & 0 & 0 & $\ldots$ \\
ST_T ECG Line Length & 2.3 & 3.5 & 1.4 & $\ldots$ \\
St slope ECG Shape & 0 & 0 & 2 & $\ldots$ \\
Num major vessels & 0 & 0 & 0 & $\ldots$ \\
Thalassemia & 1 & 2 & 2 & $\ldots$ \\
\hline
\end{tabular}

Based on Table 5 it can be explained, the data that has been preprocessed is divided into two parts, i.e. training and test data. The training data is used to build a model with a machine learning process against the dataset used. Furthermore, the test data is used to classify the class whose class is not yet known. In testing, the researcher used a test scenario such as the number of trees in the Random Forest $(\mathrm{RF})$ with the value of n_estimator = 
100, the depth level $=4$, and cross-validation value $(\mathrm{K})$ 3.2.1. Cross-validation curve

$=10$ fold. In determining the accuracy of the system from each iteration, using the most excellent accuracy. This test scenario is carried out to maximize the data used, produce a system with good performance and an accurate analysis level. These scenarios are combined in the three models used by calculating the accuracy, which will then compare the accuracy against each model to find out which model has the best performance from the Random Forest algorithm in detecting acute coronary syndrome cases. Several stages in the results and discussion, including i) classification of random forest, ii) evaluation of models, i.e. curve of cross-validation and learning, and iii) the testing and result in analysis, i.e. results from testing of the correlation matrix and precision and recall.

\subsection{Random Forest Classification}

The determine the many trees' parameters (n_estimator) to be built and the depth level in the random forest model initiation stage. After successful model initiation, the data that has been separated as training data will be entered into the model and will be trained by the model that has been built. In this study, the random forest model made has a depth of 4, and 100 trees were built. After the random forest model was created, training data would be entered to be trained into the model using the fit function. Then we will provide an iteration to describe the accuracy of the many n_estimators in the random forest model. The accuracy results for the loop on each data split can be seen in the table. The accuracy loop $\mathrm{n} \_$estimator is shown in Table 5.

Table 5. The accuracy loop n-estimator

\begin{tabular}{cc}
\hline N-Estimator & Accuracy Value's Range \\
\hline $70 \%$ & $79 \%-84 \%$ \\
$80 \%$ & $75 \%-83 \%$ \\
$90 \%$ & $60 \%-77 \%$ \\
\hline
\end{tabular}

Based on Table 5 it can be explained, that the highest accuracy is owned by the model with a split ratio of $70 \%$ (70:30) with the value ranging from $79 \%$ to $84 \%$.

\subsection{Model Evaluation}

At this stage, the providing a visualization of the performance evaluation of the model that has been produced. Creating a validation curve is one way to assess the initial effectiveness. The validation curve is a plot (graph) that shows how the model's performance responds to changes in hyperparameter values. The graph shows both training data in blue stripes and validation data in green lines. Score validation allows us to infer how the model will respond to unseen data. The hyperparameters used this time are each tree's depth (max depth) and the cross-validation of 10 folds. The evaluation for the models was carried out through crossvalidation curves and learning curves.

The validation for the random forest classifier is curved. The cross-validation curve is shown in Figure 6.

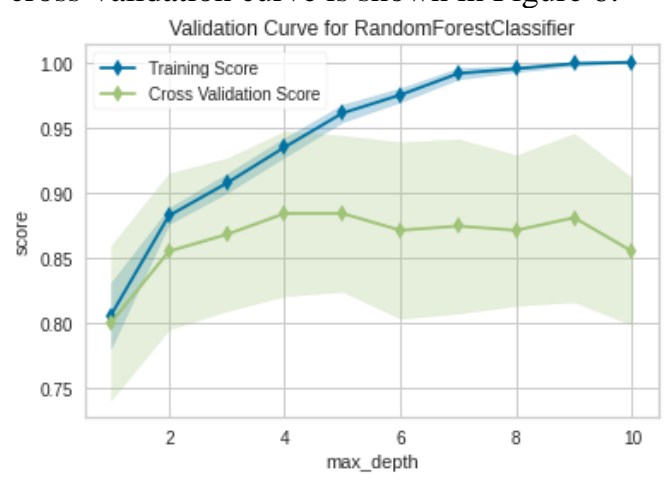

\#a) for split data of $70 \%$

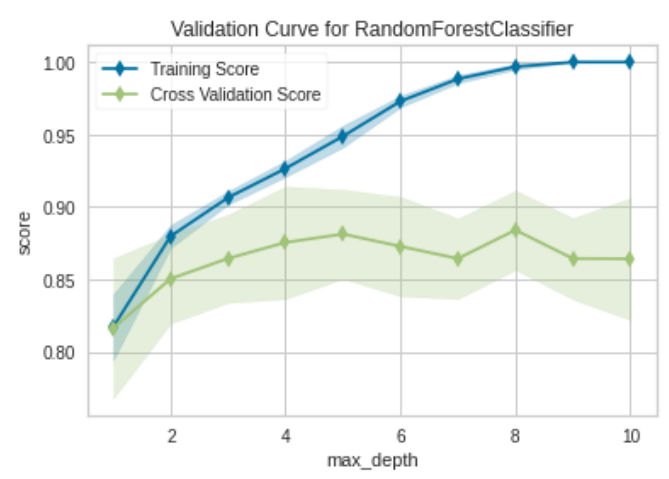

\#b) for split data of $80 \%$

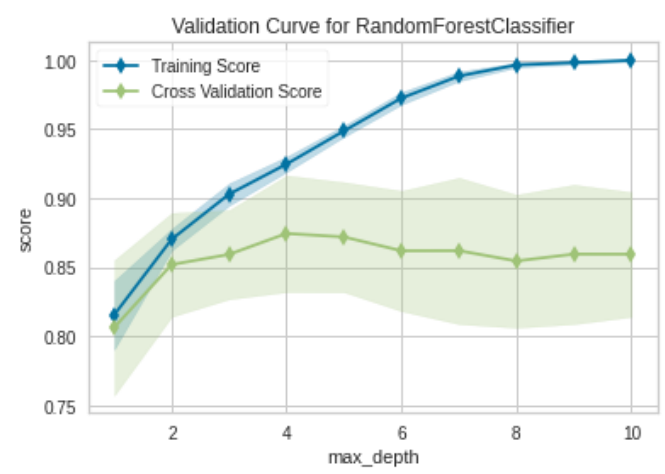

\#c) for split data of $90 \%$

Figure 6. The cross-validation curve

Based on Figure 6, shows that the resulting models' performance with a split ratio of (i) $70 \%$ has a crossvalidation accuracy range from $80 \%$ to $89 \%$, (ii) $80 \%$ has a cross-validation accuracy range from $83 \%$ to $88 \%$, and (iii) $90 \%$ has a cross-validation accuracy range from $81 \%$ to $86 \%$. As looking at the resulting curve movement patterns in each model, it can be seen that the deeper the tree level the more complex the model is, so the accuracy tends to decrease. 
Eka Pandu Cynthia, M. Afif Rizky A., Alwis Nazir, Fadhilah Syafria RESTI Journal (System Engineering and Information Technology) Vol. 5 No. 2 (2021) 369 - 378

\subsubsection{Learning curve}

The learning curve is a plot that describes the model's learning pattern of the data that is the input value and will read the pattern from the data so that it can produce a decision. The learning curve is shown in Figure 7.

Based on Figure 7, shows that the resulting model's performance with a split ratio of (i) $70 \%$ gives a learning accuracy range from $78 \%$ to $85 \%$, (ii) $80 \%$ has a crossvalidation accuracy range from $75 \%$ to $86 \%$, and (iii) $90 \%$ has a cross-validation accuracy range from $82 \%$ to $86 \%$. As looking at the training and learning data scores marked with a blue line, while the cross-validation data is marked with a green line, it can be seen the crossvalidation score increases, it shows that the model with the owned data has a good performance.

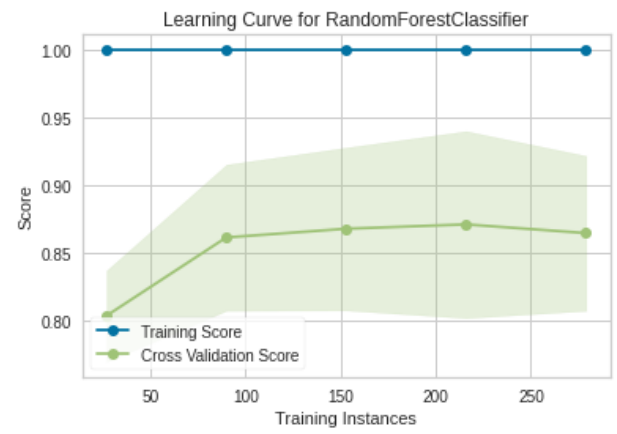

\#a) for split data of $70 \%$

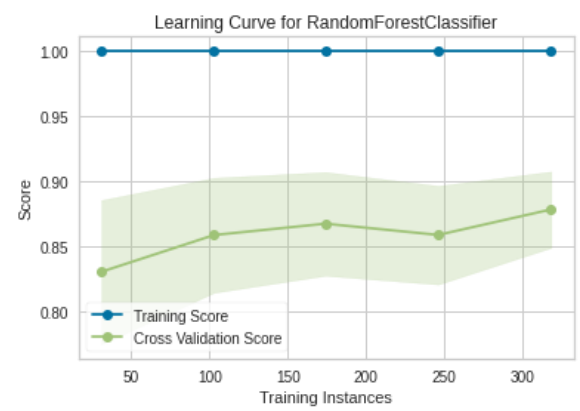

\#b) for split data of $80 \%$

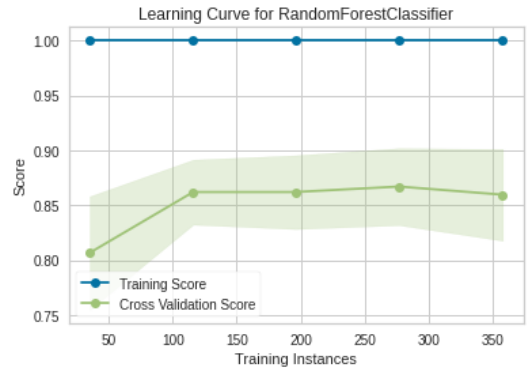

\#c) for split data of $90 \%$

Figure 7. The learning curve

\subsection{Testing and Result in the Analysis}

The explanation for the testing and result in the analysis was carried out through the result of correlation matrix testing and the results of precision and recall testing.

\subsubsection{Correlation matrix testing result}

This test is done by providing the confusion matrix visualization results to get the random forest algorithm model's overall accuracy or performance. The process of calculating confusion matrix accuracy on each split data can be seen in the table. The confusion matrix accuracy calculation is shown in Table 6.

Table 6. The confusion matrix accuracy calculation

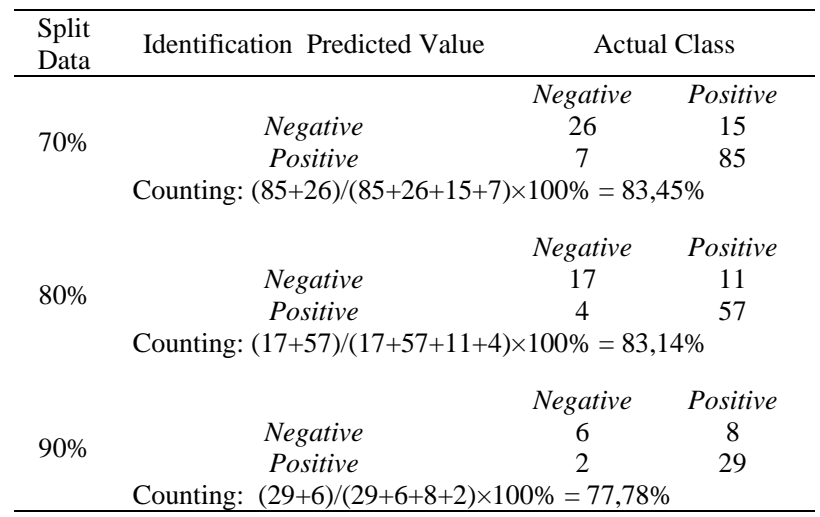

Based on Table 7 it can be explained, that there is the visualizing of the correlation matrix test with the confusion matrix. The visualization of the testing correlation matrix is shown in Figure 8.

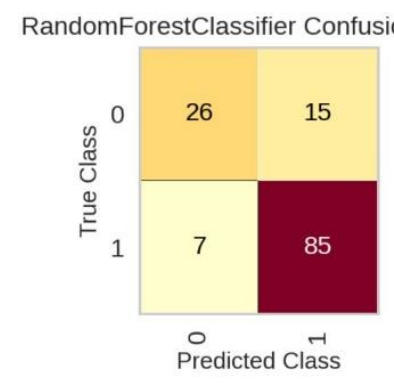

\#a) for Split Data of $70 \%$

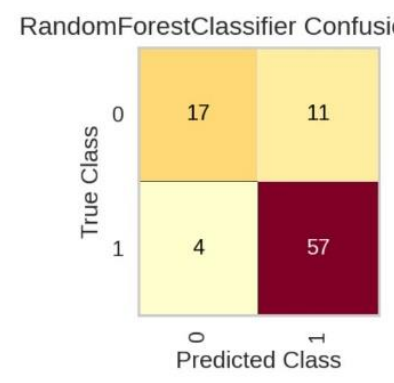

\#b) for Split Data of $80 \%$
RandomForestClassifier Confusion Matrix

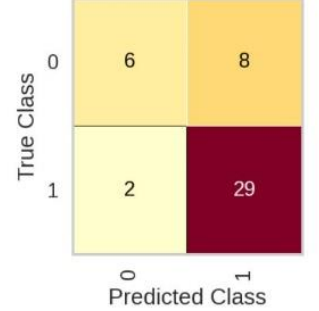

\#c) for Split Data of $90 \%$

Figure 8 . The visualization of the testing correlation matrix

DOI: https://doi.org/10.29207/resti.v5i2.3000

Creative Commons Attribution 4.0 International License (CC BY 4.0) 
Based on Figure 8 it can be explained, that the results of Meanwhile, the process of counting the accuracy of the visualization of split data of $70 \%$ model for testing recall on each split data can be seen in the table. The using the confusion matrix has the highest accuracy recall counting is shown in Table 8. value of $83.14 \%$.

\subsubsection{The Results of precision and recall testing}

The process of counting the precision accuracy for each split data can be seen in the table. The precision counting is shown in Table 7.

Table 7. The precision counting

\begin{tabular}{ccc}
\hline Split Model & Precision Counting $=\mathrm{TP} /(\mathrm{TP}+\mathrm{FP})$ & Result \\
\hline $70 \%$ & $85 /(85+15)$ & $85,00 \%$ \\
$80 \%$ & $57 /(57+11)$ & $83,82 \%$ \\
$90 \%$ & $29 /(29+8)$ & $78,38 \%$ \\
\hline
\end{tabular}

Based on Table 7 it can be explained, that the split data of $70 \%$ has the highest accuracy value of $85 \%$.

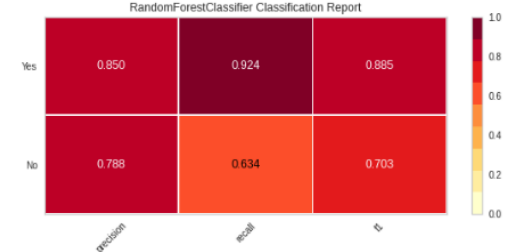

\#a) for split data of $70 \%$

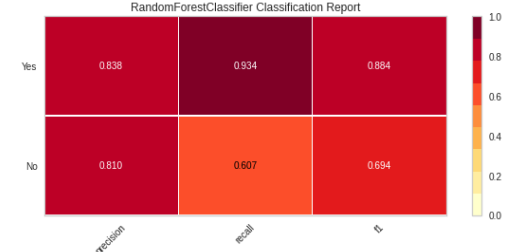

\#b) for split data of $80 \%$

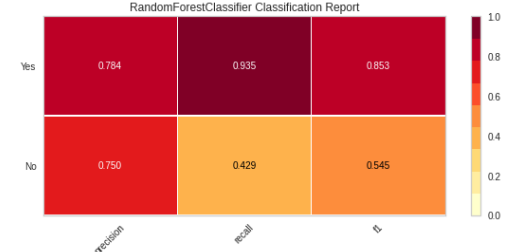

\#c) for split data of $90 \%$

Figure 9. The classification random

Based on Figure 9 it can be explained, that the results of each split ratio from $80 \%, 70 \%$, and $90 \%$ on an average the visualization that the precision and recall of the range of $80 \%$ to $89 \%$.

models built with a predetermined split ratio give results that are following the manual calculations that have been Sixth, for each model, the split ratio of $80 \%$ is $83.82 \%$, done previously.

\section{Conclusion}

The conclusion from modeling using the random forest algorithm for cases of the acute coronary syndrome, namely: First, in confusion matrix testing, the results of the overall accuracy or performance of the resulting model with split ratios of $80 \%, 70 \%$, and $90 \%$ respectively, namely $83.14 \%, 83.45 \%$, and $77.78 \%$ with the highest accuracy owned by the model with a split ratio of $70 \%$ (70:30). Second, in a random forest, the number of trees formed (n_estimator) dramatically affects the model's performance because theoretically, the more trees, the better.

Third, based on observations of the resulting accuracy in the iteration of the testing data, each number of trees and trees' addition can provide different accuracy but tend to be more stable. Fourth, based on observations made on the validation curve, with hyperparameters, namely max_depth and ten-fold cross-validation, the deeper the tree formed will result in a more complex model so that the accuracy tends to decrease. Fifth, based on observations made on the learning curve with crossvalidation of 10 fold, the model produces an accuracy of for the model with a $70 \%$ split has a precision of $85 \%$ and for the model with a split ratio of $90 \%$ produces a precision of $78.37 \%$ with the highest precision result is owned by the model. with a split ratio of $70 \%$ with a precision of $85 \%$. Seventh, in the recall test of the split ratio model, $80 \%$ has a recall of $93.34 \%$, and in the split model, $70 \%$ has a recall of $92.24 \%$ then the model with a split ratio of $90 \%$ produces a recall of $93.35 \%$ with the highest recall. They are owned by a model with a split ratio of $90 \%$. And eighth, when viewed from the classification report results, the ratio that better recognizes each class with the highest level of balance seen from the ratio of precision and recall and the accuracy of each target is a model with a split ratio of $70 \%$ (70:30) with a precision of $85 \%$, a recall of $92.24 \%$ and an accuracy of $83.45 \%$.

For further research, it can be suggested to use optimization in the random forest algorithm, especially to overcome the over-fitting or under-fitting of the resulting model.

\section{References}

[1] Z. Masetic and A. Subasi, "Congestive Heart Failure Detection Using Random Forest Classifier," Comput. Methods Programs Biomed., vol. 130, pp. 54-64, 2016, doi: 10.1016/j.cmpb.2016.03.020. 
Eka Pandu Cynthia, M. Afif Rizky A., Alwis Nazir, Fadhilah Syafria

RESTI Journal (System Engineering and Information Technology) Vol. 5 No. 2 (2021) 369 - 378

[2] T. I. Rohan, Awan-Ur-Rahman, A. B. Siddik, M. Islam, and M. S. U. Yusuf, "A Precise Breast Cancer Detection Approach Using Ensemble of Random Forest with AdaBoost," in 5th International Conference on Computer, Communication, Chemical, Materials and Electronic Engineering, IC4ME2 2019, 2019, no. June 2020, pp. 10-14, doi: 10.1109/IC4ME247184.2019.9036697.

[3] H. Byeon, "Is the Random Forest Algorithm Suitable for Predicting Parkinson's Disease with Mild Cognitive Impairment Out of Parkinson's Disease with Normal Cognition?," Int. J. Environ. Res. Public Health, vol. 17, no. 7, pp. 1-14, 2020, doi: 10.3390/ijerph17072594.

[4] A. M. Antoniadi, M. Galvin, M. Heverin, O. Hardiman, and C. Mooney, "Prediction of Caregiver Burden in Amyotrophic [18] Lateral Sclerosis: A Machine Learning Approach Using Random Forests Applied to A Cohort Study," BMJ Open, vol. 10, no. 2, pp. 1-8, 2020, doi: 10.1136/bmjopen-2019-033109.

[5] K. K. Chari, M. Chinna Babu, and S. Kodati, "Classification of Diabetes Using Random Forest with Feature Selection Algorithm," Int. J. Innov. Technol. Explor. Eng., vol. 9, no. 1, pp. 1295-1300, 2019, doi: 10.35940/ijitee.L3595.119119.

[6] B. Dai, R. C. Chen, S. Z. Zhu, and W. W. Zhang, "Using Random Forest Algorithm for Breast Cancer Diagnosis," in International Symposium on Computer, Consumer and Control [20] (IS3C), 2018, pp. 449-452, doi: 10.1109/IS3C.2018.00119.

[7] C. Iwendi et al., "COVID-19 Patient Health Prediction Using Boosted Random Forest Algorithm," Front. Public Heal., vol. 8, no. July, pp. 1-9, 2020, doi: 10.3389/fpubh.2020.00357.

[8] M. Z. Alam, M. S. Rahman, and M. S. Rahman, "A Random Forest Based Predictor for Medical Data Classification Using Feature Ranking," Informatics Med., vol. 15, no. April, pp. 112, 2019, doi: 10.1016/j.imu.2019.100180.

[9] P. Kaur, R. Kumar, and M. Kumar, "A Healthcare Monitoring System Using Random Forest and Internet of Things (IoT)," Multimed. Tools Appl., vol. 78, no. 14, pp. 19905-19916, 2019, doi: 10.1007/s11042-019-7327-8.

[10] J. Ricordeau and J. Lacaille, "Application of Random Forests To Engine," in International Congress of the Aeronautical Sciences (ICAS), 2010, no. April, pp. 1-10.

[11] K. Kumar, "Health Care Analysis Using Random Forest Algorithm," J. Chem. Pharm. Sci., vol. 10, no. 3, pp. 13591361, 2017.

[12] O. Pauly, "Random Forests for Medical Applications,"
Technischen Universität München, 2012.

[13] L. Pierson, Data Science for Dummies, 2nd ed. New Jersey: John Wiley \& Sons Inc., 2017.

[14] Y. Liu, Python Machine Learning By Example - Second Edition, 1st ed. Birmingham: Packt Publishing, 2017.

[15] P. Mathur, Machine Learning Applications Using Python, 1st ed. Karnataka: Apress Media LLC, 2019.

16] M. Kubat, An Introduction to Machine Learning, 2nd ed. Switzerland: Springer International Publishing AG, 2017.

[17] E. Corbett, "The Real-World Benefits of Machine Learning in Healthcare," 2017. https:/www.healthcatalyst.com/clinicalapplications-of-machine-learning-in-healthcare (accessed Mar. 06, 2021).

[18] W. H. Organization, "Cardiovascular diseases," 2017. https://www.who.int/en/news-room/fact-

sheets/detail/cardiovascular-diseases-(cvds) (accessed Mar. 27, 2021).

[19] K. Akyol, E. Çalik, Ş. Bayir, B. Şen, and A. Çavuşołlu, "Analysis of Demographic Characteristics Creating Coronary Artery Disease Susceptibility Using Random Forests Classifier," in International Conference on Soft Computing and Software Engineering (SCSE), 2015, vol. 62, pp. 39-46, doi: 10.1016/j.procs.2015.08.407.

[20] I. Yekkala and S. Dixit, "Prediction of Heart Disease Using Random Forest and Rough Set Based Feature Selection," Int. J. Big Data Anal. Healthc., vol. 3, no. 1, pp. 1-12, 2018, doi: 10.4018/ijbdah.2018010101.

[21] R. Ani, A. Augustine, N. C. Akhil, and O. S. Deepa, "Random Forest Ensemble Classifier to Predict the Coronary Heart Disease Using Risk Factors," in International Conference on Soft Computing System, Advances in Intelligent Systems and Computing, 2016, vol. 397, pp. 701-710, doi: 10.1007/978-81322-2671-0_66.

[22] S. Polamuri, "How The Random Forest Algorithm Works in Machine Learning," 2017. https://dataaspirant.com/randomforest-algorithm-machine-learing/ (accessed Mar. 06, 2021).

[23] J. Novakovic, A. Veljovi, S. Iiic, Z. Papic, and M. Tomovic, "Evaluation of Classification Models in Machine Learning," Theory Appl. Math. Comput. Sci., vol. 7, no. 1, pp. 39-46, 2017, [Online]. Available: https://uav.ro/applications/se/journal/index.php/TAMCS/articl e/view/158.

DOI: https://doi.org/10.29207/resti.v5i2.3000 